\title{
Hypergraph Model of Digital Topology for Grey Level Images *
}

\author{
A. Bretto \\ TSI CNTS URA 842 \\ Ingénierie de la vision \\ Site G.I.A.T Industries \\ 3, rue Javelin Pagnon \\ 42007 Saint-Etienne \\ cedex 1 FRANCE \\ bretto@univ-st-etienne.fr
}

\author{
S. Ubéda \\ LIP CNRS URA 1398 \\ ENS-Lyon \\ 46, allée d'Italie \\ 69364 Lyon \\ Cedex 7 FRANCE \\ ubeda@lip.ens-lyon.fr
}

\begin{abstract}
This paper gives a hypergraph based theoretical approach of digital topology. The study of digital topology is in connection with most image processing research. An application of hypergraph based digital topology to grey level images segmentation is presented.

The originality of this work comes from the introduction of a new topology concept based on hypergraphs but above all from the possibility to modelize grey level images as well as colored images.

The model is based on the fundamental Helly property of Hypergraphs. This paper introduces the Helly filter which gives to the neighborhood hypergraph associated with an image, the Helly property. As an application example, the model is successfully used to build a segmentation process.
\end{abstract}

\section{Introduction}

The only sets which can be handled on computers are discrete or digital sets. As presented in [4], there are almost two sources for discrete sets:

- standard data structures of computer sciences,

- discretization of continuous objets.

In the field of image processing, the image is acquired by spatially resolved measurement of physical parameters characterizing the objects. We deal with

*This work have been supported by the project "Nouvelles Architectures et Développement d'application", Région Rhône-Alpes. 
intensity images in the case the result of acquisition is expressed by a single value at each location of measurement. The image is then given as an ordered set of intensity values forming a grid of $\mathbb{Z}^{2}$.

In digital topology applied to image processing, graph structures on the set $\mathbb{Z}^{2}$ are usually considered and called adjacency graphs. The two points $(i, j)$ and $\left(i^{\prime}, j^{\prime}\right)$ of $\mathbb{Z}^{2}$ are said to be 8 -adjacent if they are distinct and satisfy $\max \left(\left|i-i^{\prime}\right|,\left|j-j^{\prime}\right|\right)=1$. They are said to be 4 -adjacent if they are 8 -adajcent and have one of their coordinates in common. These two adjacency relations provide $\mathbb{Z}^{2}$ with two non oriented graph structures.

An image can be considered as a set of objects organised in a scene. As combinatorics is a science studying the organisation of elements which can be mathematically formalized, we are naturally led to modelize an image by a combinatorial structure, that is to say neighborhood hypergraphs in this case. Starting with the 8-adjacency graph structure we propose a modelization of a grey level image and derive from such a modelization a new segmentation algorithm.

Section 2 presents some general ideas about hypergraphs and the hypergraphs associated with grey level images. Most of the results in hypergraph theory are based on hypergraphs having the Helly property. In this section we deal with a complete study of hypergraphs associated with an image according to this property. Section 3 presents a filter algorithm named the Helly Filter that has to be applied to the image in order to obtain an associated hypergraph having the Helly property. Section 4 presents a new segmentation algorithm based on the associated hypergraph. This segmentation algorithm is a new one, but it falls in the class of region growing segmentation technics. It is very similar to the segmentation algorithm presented in [7]. Therefore, the hypergraph model gives a new validity to this kind of approach and can be used to prove some properties of various image processing kernels. Section 5 gives a conclusion and some consideration for further development of the hypergraph based approach.

\section{Hypergraph applied to digital topology}

The idea of studying families of sets as generalized graphs appeared in the sixties. This vision gave rise to the theory of hypergraphs, a theory which has proved to be an extremely important tool in different fields of human activities such as psychology, operational research, scheduling etc ... $[1,5]$.

One of the main categories of hypergraphs is the category of neighborhood hypergraphs. The elements of this family are generated thanks to graphs. The notion of adjacency in discrete topology is modelized by graphs. In the following part of this article, we suggest a new way of modelizing; the notion of hypergraph enables us to modelize not only the notion of adjacency but also the notion of neighborhood which is thus no longer solely binary. 


\subsection{Hypergraph}

In the following section, we summarize some notions of the hypergraph theory useful for understanding the modelization presented afterwards.

Definition 1 Let $X=\left\{x_{1}, x_{2}, \ldots, x_{n}\right\}$ a set, a hypergraph defined on $X$ (the set of vertices) is a family $E=\left\{E_{1}, E_{2}, \ldots, E_{n}\right\}$ of subsets of $X$ called hyperedges, with :

$$
E_{i} \neq \emptyset \quad \forall i \in\{1, \ldots, n\} \text { and } \bigcup_{i \in\{1, \ldots, n\}} E_{i}=\mathbf{X}
$$

Let us note :

$$
H(X, E)
$$

Definition 2 Let us consider an hypergraph $H(X, E) . W \subset E$ is an intersecting family of $H$ if and only if $\forall E_{i}, E_{j} \in W, E_{i} \cap E_{j} \neq \emptyset$.

Definition 3 Let us consider an intersecting family $W$ of $H$. $W$ is a star centered in $x \in X$ if and only if

$$
W=\left\{E_{i} \mid x \in E_{i}\right\}
$$

The degree of $x$ is the cardinality of star centered in $x$.

Figure 1 shows two examples of intersecting hyperedges.

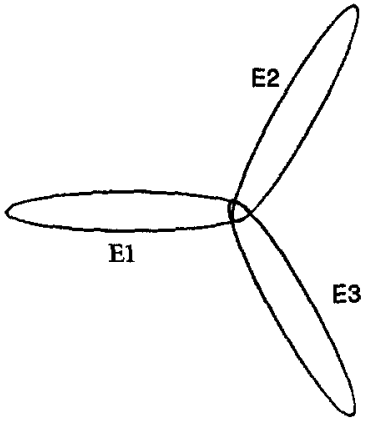

(a) star

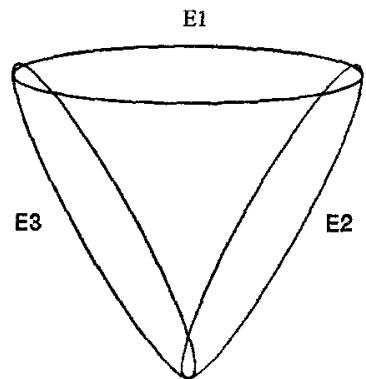

(b) triangle

Figure 1: Family of three hyperedges, (a) intersecting two by two with a nonempty intersection, (b), intersecting two by two with an empty intersection.

An important property on the hypergraph theory is the Helly property :

Definition 4 If any intersecting family of $H(X, E)$ is a star, $H$ has the Helly property. 
Definition 5 Let us consider a loopless graph $G(X, U)$, where $X$ represents the set of vertices and $U$ represents the set of edges. The Hypergraph $H_{G}$ associated with $G$ is the hypergraph defined on $X$ where hyperedges are defined by:

$$
\forall x \in X, E_{x}=x \cup\{y \in X,(x, y) \in U\}
$$

The class of hypergraphs associated with a graph are called neighborhood hypergraphs.

\subsection{Hypergraphs associated with an image}

A picture is digitalized by sampling its brightness values on a discrete grid of points [9]. These points are called pixels. The grid used here is the 8-connected grid $[10,11]$. So that a grey level image is a function defined on $X \subseteq \mathbb{Z}^{2}$ with values in $\mathbb{Z}^{2}$.

Let us define $V_{8}(x)$ the 8 neighborhood of the pixel $x=\left(x_{1}, x_{2}\right)$ as usual:

$$
V_{8}(x)=\left\{y=\left(y_{1}, y_{2}\right) \in \mathbb{Z}^{2}, \max \left(\left|x_{1}-y_{1}\right|,\left|x_{2}-y_{2}\right|\right)=1\right\}
$$

We call $\Gamma$ a neighborhood relation on an image, and $I$ the application on $X$ with values in $P(X)$ defined by:

$$
\Gamma_{\alpha}(x)=\left\{y \in V_{8}(x),|I(x)-I(y)| \leq \alpha\right\}
$$

where $I(x)$ is the grey level of pixel $x$ and $\alpha$ is a constant.

This neighborhood relation on the image $I$ forms a graph, to which we can associate a neighborhood hypergraph defined by :

$$
H_{\alpha}(I)=\left(X,\left(x \cup \Gamma_{\alpha}(x)\right)\right)
$$

Note that for each value of the constant $\alpha$, there exists a different neighborhood graph to which a different neighborhood hypergraph is associated. Such hypergraphs are said to be associated with the image $I$ in accordance with the constant $\alpha$; reciprocally, given neighborhood hypergraph $H$ whose vertices are valued in $\mathbb{Z}$ and a constant $\alpha$, there exists a unique discrete image $I$ such as $H=H_{\alpha}(I)$.

\section{Helly filter}

Our model of images is based on the fact that the hypergraph associated with an image may have the Helly property. Unfortunately this is not always true. Therefore, we know that the hypergraph associated with the 8-connected grid has the Helly property [3] (this hypergraph is obtained with $\alpha=+\infty$ ). This condition is necessary but not sufficient to ensure that the hypergraph associated with the image has this property. We use this result to build an algorithm which associates the image $I^{\prime}$ to the image $I$ so that the hypergraph $H_{\alpha}^{\prime}\left(I^{\prime}\right)$ has the Helly property and no visual difference can be seen between $I$ and $I^{\prime}$. 
The Helly property is an uniformity notion yet sharp contours and noises could cancel the property. Therefore, this condition allows us to build a kind of "filter" process that corrects the little local configurations of pixels that cancel the Helly property. The following algorithm is named the Helly Filter and this algorithm restores the Helly property to the hypergraph associated with the image. This filter only causes some little alterations of grey levels for a limited number of configurations in the image. The next section presents a segmentation process taking as input this "ideal" filtered image (see Fig. 2).

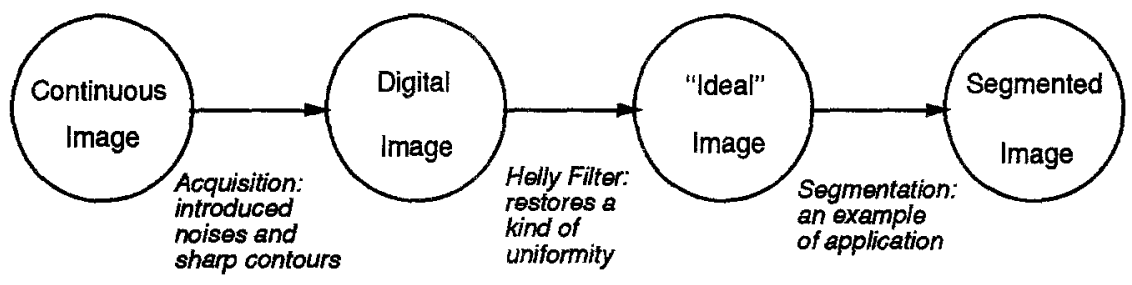

Figure 2: sketch of the application.

Step 1 Let $x$ be an element in the domain of $I . x \cup \Gamma_{\alpha}(x)$ are the edges generated by $x$ in $H_{\alpha}(I)$.

Step 2 Let $A_{x}$ be the set of edges of $H_{\alpha}$ associated with the vertices of the neighborhood of $x$, we have:

$$
A_{x}=\bigcup_{y \in V_{B}(x)}\left(y \cup \Gamma_{\alpha}(y)\right) .
$$

We search in $P\left(A_{x}\right)$ for the set of intersecting families.

- We build the graph $G I_{x}$ of the intersections of the edges $A_{x}$ defined by :

- the set of vertices $V=G(x)$

- the set of edges $E$ defined by $\left(y_{1}, y_{2}\right) \in E$ if and only if

$$
\left(y_{1} \cup \Gamma_{\alpha}\left(y_{1}\right)\right) \cap\left(y_{2} \cup \Gamma_{\alpha}\left(y_{2}\right)\right) \neq \emptyset
$$

Step 3 We search for the set of the maximal intersecting families of $A_{x}$.

- We search for the complete maximal sub-graphs of the graph $G I_{x}$.

Step 4 Let $F_{y_{1}}, F_{y_{2}}, \ldots, F_{y_{l}}$ be the set of the maximal intersecting families: (to avoid processing the same family several times, and to process every vertex, we will only deal with the families whose gravity center is $x$ ). $F_{x_{i}}$ is centered on $x$ if the gravity center of $F_{x_{i}}$ is $x$. ( $x$ is adjacent to any $F_{x_{i}}$ ).

- If $l=1$ ( there is only one family with $x$ as a center), and if $x \in F_{x_{1}}$ then $F_{x_{1}}$ is a star ( the partial sub-hypergraph has the Helly property).

- Else if $l>1$ or if $l=1$ and $x \notin F_{x_{1}}$ We build the star as following:

- Let $S_{x}=\bigcup_{i=1,2, \ldots l} F_{x_{i}}$, we test the variation of the grey level vertices of $S_{x}$. 
- If the gap is superior to $2 \alpha$, the vertex whose grey level is the farthest from the grey level of $x$, is taken off $S_{x}$. This process is repeated until the gap becomes acceptable.

- If $x$ is the center of a star whose edges belong to $S_{x}$, it is over.

Else we modify the grey level of $x$ so that $x$ is the center of $S_{x}$.

Step 5 For some vertices their grey level has changed, so we must define again the neighbourhood hypergraph associated with the image, because some edges have been modified. The same applies to intersecting families.

Note that the search for the maximal intersecting families of the graph used in step 3 involves a search for maximal clique which is well known as an NPComplet problem. Therefore, in practice, the size of the problem in our application (the size of the neighborhood) is finite and small.

Theorem 1 The Helly filter is convergeant, and if $p$ is the number of neighbors of a vertex on the grid, $p^{2}$ is an increase of the iteration number of the filter.

Proof: At the ith process of the algorithm, a vertex whose grey level has been changed previously, has its grey level changed again, if $x$ is again candidate to the gravity center of $H(x), F_{x_{p}}, \ldots \ldots, F_{x_{p+j}} .(H(x)$ is the star centered on $x$ and $F_{x_{p+j}}$ is an maximal intersecting family that can be a star.) The grey level of the neighbour of $x, y$ is changed if $y$ is a candidate to the gravity center of $H(x), H(y), F_{x_{p}}, \ldots \ldots, F_{x_{p+j}}, F_{y_{p}}, \ldots, F_{y_{p+k}}$.

- The cardinality of a star is increasing. Let $H(x)$ be a star built at the ith process (the neighborhood relation being reflexive it is sufficient to reason on $x)$.

Let $H(x), H(y), F_{x_{p}}, \ldots \ldots, F_{x_{p+j}}$, be the intersecting families at the $i+1$ ith process, any generator vertex of the edges of these families are the neighbours of $x$, thus their grey levels are at more or less from $I(x)$ and consequently will not be knocked out by building the new star. The new middle will be defined at more or less of the grey level of these vertices. $H(x)$ can only take edges in the families $F_{x_{p+j}}$.

- The number of intersecting families accepting $x$ as a gravity center, being finished and the cardinality of $H(x)$ being limited, then the convergence is proved.

- A vertex $x$, on the grid having $p$ neighbours having themselves $p$ neighbours, will have its grey level changed $p^{2}$ times at most.

This filter has been implemented and visually the filtered image $I^{\prime}$ is identical to the original image $I$ (there are only very few pixels whose grey level has changed). Note that in our application the number of neighbors is 8 , involving at most 64 iterations. This is the worst case and in practice the number of iterations needed is far from that. 


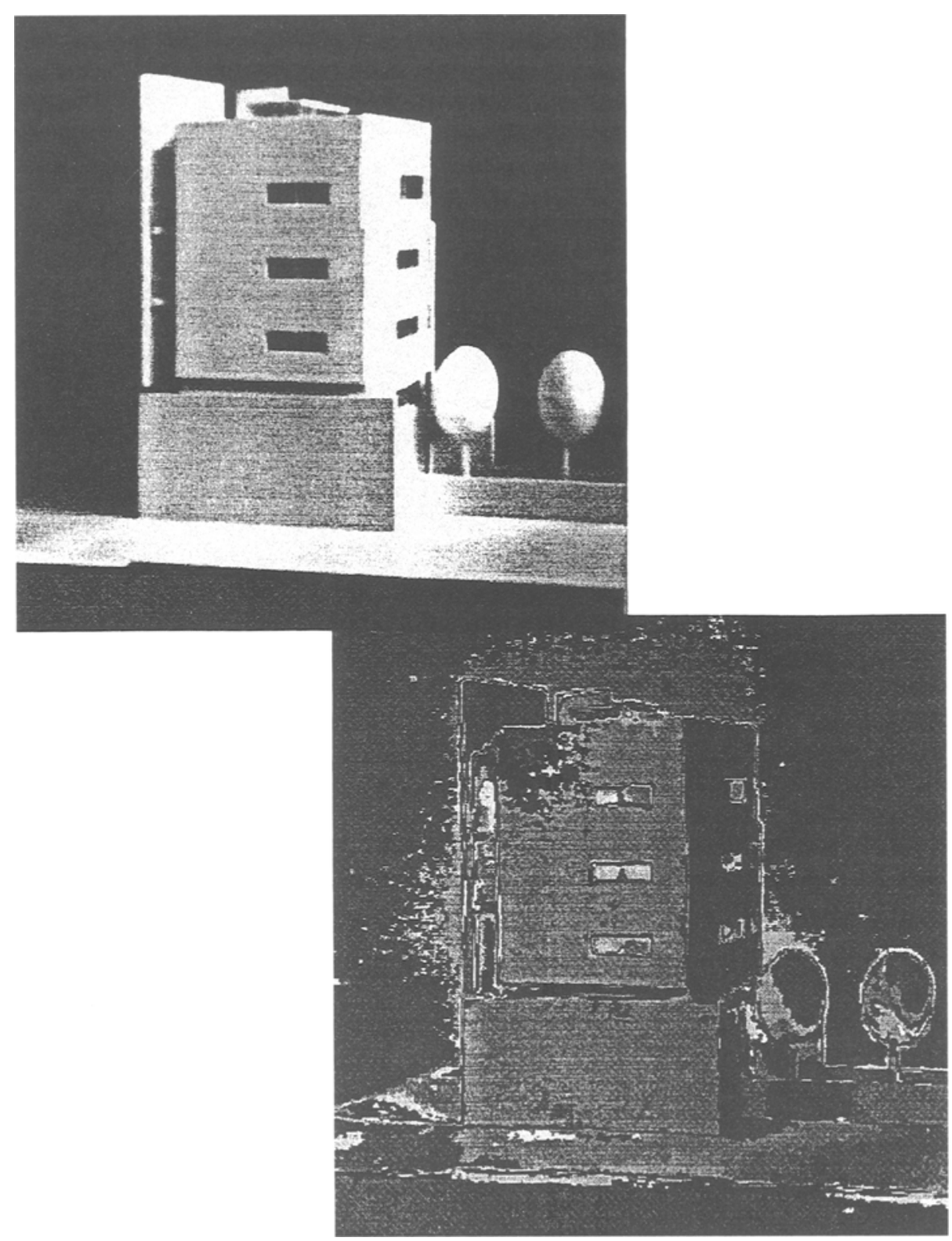

Figure 3: an image test and one possible segmentation. 


\section{Segmentation algorithm}

Segmentation is an important part of image analysis. This is a process of grouping image pixels (see [8] and [6]) from one or more characteristics. We propose to use the Helly property to give us a strategy to aggregate pixels.

Let us recall that a segmentation is a partition of $X$ in accordance with a homogeneity criterion. In the case of the hypergraph associated with an image it amounts to a vertex partition.

Obviously the Helly property is not linked to the physical formation of an image. So the first step of the segmentation process is to operate a transformation on the image such that the Helly property is satisfied. This is done by using the Helly Filter previoulsy presented.

The idea is to detect intersecting stars in the hypergraph associated with the image; the process agregates these stars when grey levels of the centers of the stars are within a small interval. Such a segmentation process falls in the class of region growing segmentation technics [8]. Similar algorithm have been presented for exemple in [2].

\section{Agregated stars algorithm}

Step 1 We do a recovery of image by a set of stars :

$$
E=\left\{e_{1}, e_{2}, \ldots, e_{n}\right\} .
$$

Step 2 Building of the star aggregating area.

We propose a color in the center of a star, of grey level $g$.

This color is propagated at any vertex of any edge of the star.

From each vertex we repeat the following process:

- The same color is propagated from the centers of the stars which cover these vertices if their grey level is within the interval $[g-\alpha ; g+\alpha]$.

- We stop when a vertex or a star center is already colored.

- We begin with color one for the center of star $e_{1}$.

This color is propagated, some vertices are colored with the same color, and if necessary we propagate the same color once again from other star centers, and so on.

Once color one is used, we search in the list of stars for the first whose center is not colored and we give it color two. This color will be propagated with the same process.

- We continue in the same way with a new color until every center of the stars has been colored.

It is not necessary to check if there are vertices which have not been colored, because by this method every vertex is covered by a star and thus each one has been colored once. 
Remark on complexity: finding the maximum Helly families covering up the image in step 1 is in $O(n)$ where $n$ is the number of pixels in the images. The partition built in step 2 is computed in a time proportional to the maximum intersecting Helly families number, which is image dependent. One exempleof a possible segmentation on a simple image is presented in Fig. 3.

\section{Conclusion}

In this paper we have presented a new model of digital images which can unify several approaches of image processing. The neighborhood hypergraph associated with an image allows us to model grey level images as well as colored images. It is based on the fundamental Helly property of hypergraphs.

The Helly property is uniformity notion and sharp contours and noises in the image could cancel the property. Therefore, we present the Helly Filter, a process that corrects the little local configurations of pixels that cancel the Helly property. This filter restores the Helly property of the hypergraph associated with the image. This filter only does some little modifications of grey levels for a limited numbers of configurations in the image.

As an application example, we introduce a new concept of segmentation based in this case on the Helly property. This algorithms falls in the region growing family technics[12].

\section{References}

[1] Berge. Hypergraphes. Authier-Villard.

[2] P. Bertolino. Contribution des pyramides irrégulières en segmentation d'images. PhD thesis, INPG, 1995.

[3] A. Bretto and B. Laget. Neighborhood hypergraph and image analysis. In Signal processing II (Fontainebleau, France), pages 62-69, 1994.

[4] U. Eckhardt and L. Latecki. Digital topology. Research report, Univ. Hambourg, Dept. of Applied Math., 1989.

[5] Gondran and Minoux. Graphs and algorithms. Wiley - Introduction Series in Discrete Math. and Optimization.

[6] R.M. Haralick and L.G.Shapiro. Survey: Image segmentation technique. Computer Vision and Image Processing, (29):100-132, 1985.

[7] A. Montanvert, P. Meer, and P. Bertolino. Hierarchical shape analysis in grey level images. In NATO Advance Research Workshop Shape in Picture (The Netherlands), 1992.

[8] N.R. Pal and S.K. Pal. A review on image segmentation techniques. Pattern Recognition, 26(9):1277-1294, 1993. 
[9] A. Rosenfeld and A. C. Kak. Digital Picture Processing. Academic Press, New-York, 1989.

[10] J. Serra. Image Analysis and Mathematical Morphology. Academy Press, 1982.

[11] J. Serra and L. Vincent. An overview of morphological filtering. circuits systems signal process, 11(1), 1992.

[12] S.W. Zucker. Survey region growing: Childhood and adolescence. Computer Vision, Graphics and Image Processing, (5):382-399, 1976. 